\title{
DESIGN OF PORTABLE INSTRUMENT FOR MEASURING AGRICULTURE FIELD SIZE BASED ON GPS
}

\author{
Xinjian Xiang, Chunting Yang \\ College of Automation \& Electrical Engineering, Zhejiang University of Science and Technology, \\ Hangzhou, 310023, P. R. China, Tel: +86-571-85070268,Email: hzxxj@sina.com
}

Abstract: Global Positioning System (GPS) plays an important role in precision agriculture. Applying positioning function of GPS, this paper researches on portable instrument for measuring field size. Instrument consists of low-cost GPS module, data acquisition board in microcontroller, large capacity EEPROM storage, RS232C communication interface, keyboard and LCD display. GPS position information acquisition technology, method for calculating polygonal fields geometric size, software and hardware design principles of instrument, GPS measuring error analysis and accuracy control of instrument are provided. Experiment results prove that measuring instrument can fast measure geometric size of fields in any shape, relative measuring error is less than $2.5 \%$, and the bigger the size of field is, the higher the measuring accuracy is.

Keywords: GPS, field geometric size, measuring instrument, precision agriculture

\section{INTRODUCTION}

Field geometric size is one of the most basic data. Field geometric size such as distance, perimeter and area directly decides the input amount of seeds, fertilizer, pesticides and other production materials. It is also the main basis for calculating working time and charging when agriculture machine are doing field working. Traditionally, geometric size of fields are most measured by tape, simple and practical. However, it is only suitable for small 
size field, for which with large are, it cost lots of time and effort, and easily causes mistakes. Also, it is difficult to measure the perimeter and area of irregular fields. As the core technology of supporting precision agriculture (Zhang et al., 2002), GPS global satellites positioning system can provide real-time navigation information such as longitude, latitude and elevation etc. With GPS positioning function, each point's coordinate in field can be calculated, which makes it possible to calculate fields distance, perimeter and area with mathematic methods. Adopting low-cost GPS module, this paper develops portable instrument for measuring field geometric size, realizing outdoor fast GPS positioning measurement for regular or irregular fields, green land, forest, water area and tidal flat in any shape. For seeds, fertilizer and pesticides input, total amount can be calculated by inputting single operation amount. For machine operation, operation single price input, and instrument directly displays the charge. Large capacity EEPROM storage, RS232C communication interface, graphic LCD can input measuring data into computer and display outline of measured field and manage field data base. Instrument has functions of storing, displaying and printing outline graphic. Additionally there are two big functions: for conveniently using the instrument, there is additional manual measuring for geometric size (for relatively regular district or inconvenient passing area, such as building, bridge, big tree and other obstacles, manual measurement can be taken. Only apex data are needed, not necessarily to walk around); and data filling up (when measuring area, if end and starting points not meet, instrument will automatically filling data with the straight line between them and calculate the area).

\section{GPS POSITIONING INFORMATION ACQUISITION}

By GPS receiver, GPS positioning information is acquired. Commonly used GPS receiver can be divided into measuring, navigation, handwriting SMS, OEM board and many kinds. There is big difference in functions and prices among them. For example, differential GPS receiver Ag GPS132 produced by American company Trimble has positioning accuracy with 10-2 meter level. It is widely used in precision agriculture. However, because of its high price, large volume and high power consumption, it is not suitable for portable instrument. This research takes GPS 25LP OEM board made by GARMIN Company to position. GARMIN Company is global GPS OEM 
board supplier. 25LP GPS OEM board is single point mode product, whose main function is: parallel 12 channels receiving; recapture time $<2$ s, hot starting time is $15 \mathrm{~s}$, cold starting is $45 \mathrm{~s}$, automatically searching time is $90 \mathrm{~s}$; positioning accuracy is $15 \mathrm{~m}$, in differential (DGPS) situation, positioning accuracy $<5 \mathrm{~m}$; external antenna is provide to help receive information; volume is small and power consumption low; $5 \mathrm{~V}$ power source supplier.

GPS OEM board's input and output sentence accords serial communication protocol. Default communication baud rate is $4800 \mathrm{bps}$, data structure is 8 data bits, 1 initial bit and 1 stop bit, no even-odd check bit, initialization of output data format is NMEA-0183.NMEA-0183 is the standard format for marine electronic device designed by American Ocean Electronic Association. As the format is ASCII code string, direct and easily processed, it can be directly judged and separated in many advanced languages to acquire data users need. Through input sentences, users initialize GPS OEM board, set data format, communication baud rate, required output categories; output sentence is to output various data GPS information to users.

In fields geometric size positioning measurement, \$ GPGGA sentence format recommended is taken. \$ GPGGA sentence contain commonly used positioning information as longitude, latitude, speed, date, time, receiver condition. This sentence consist of 75 characters, each data segment has a fixed position. For example, position of longitude data is bit 19 to bit 27 in the string, position of latitude data is bit 31 to 40 . Reading relative district data, positioning information is acquired. Then longitude and latitude strings are transformed into numbers, further the distance, perimeter and area are calculated.

GPGGA sentence is the standard data format output by GPS OEM of GARMIN, starting with character "\$", ending with carriage return character, data segments and checksum in the middle and comma separating data segments. For instance, if real-time receiving sentence "\$GPGGA": \$GPGGA, 114641,3002.3232,N, 12206.1157,E, 1,03, 12.9,53.2,M,11.6,M, *4A. This GPS positioning data information sentence means: UTC Time is $11 \mathrm{~h} 46 \mathrm{~m} 41 \mathrm{~s}$, locates at north longitude $30^{\circ} 2.3232^{\prime}$, east latitude $122^{\circ}$ 6.1157', common GPS positioning method, receiving 3 satellites, horizontal accuracy is $12.9 \mathrm{~m}$, the height of antenna from the sea level is $53.2 \mathrm{~m}$, location is $11.6 \mathrm{~m}$ higher than horizon, checksum is $4 \mathrm{AH}$. As GPS OEM sends more than 1 sentence, so to completely receive this "\$GPGGA" sentence, it is necessary to judge the head of the sentence, that is 7 characters of "\$GPGGA". When these 7 characters are completely received, the data can be ensured useful. 


\section{GEOMETRIC SIZE CALCULATION METHOD}

Walking around the field with GPS receiver, a group of positioning data consisting of many points can be measured. According to the order of time they are: $(\mathrm{X} 1, \mathrm{Y} 1),(\mathrm{X} 2, \mathrm{Y} 2) \ldots(\mathrm{Xn}, \mathrm{Yn})$, of which $\mathrm{X}$ is longitude, $\mathrm{Y}$ latitude. Connecting these points, then polygon with $\mathrm{n}$ apexes is constructed, as shown in Figure 1.

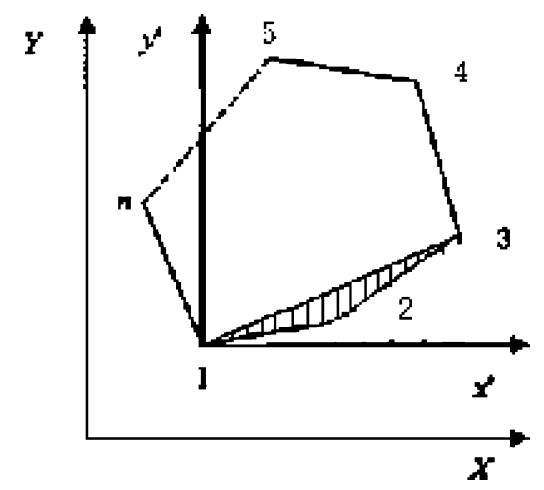

Figure 1. Area calculation for polygon field

As earth is ellipsoid, to accurately calculate distance or area, generally they are transformed into plane coordinate by projecting. In our country, for big size map, Gauss-Krüger projecting is used (Zhu et al., 2000). However, projecting calculation is very complicated and hardly being realized in microcontroller. To simplify calculation, we regard earth as pellet. As the field distance is short, generally less than $1 \mathrm{~km}$, and area small, so the error brought by simplification can be neglected. Taking earth radius as $6371116 \mathrm{~m}$, then longitude and latitude can be transformed into plane coordinates:

$$
x=2 \pi R X / 360, y=2 \pi R Y \cos Y / 360
$$

In above equation: $\mathrm{R}$ is the earth radius, $\mathrm{X}$ longitude/m, $\mathrm{Y}$ latitude/m. As $\mathrm{x}, \mathrm{y}$ values are big, if used to calculate area, big error will be caused. So the first point is set as the original point of coordinate, new coordinate is made by relative displacement, shown as Figure 1:

$$
\begin{aligned}
& x_{i}^{\prime}=x_{i}-x_{1} \\
& y_{i}^{\prime}=y_{i}-y_{1}
\end{aligned}
$$

Then distance between two adjacent points is:

$$
L_{i}=\sqrt{\left(x_{i+1}^{\prime}-x_{i}^{\prime}\right)^{2}+\left(y_{i+1}^{\prime}-y_{i}^{\prime}\right)^{2}}
$$

Perimeter of polygon is: 


$$
G=\sum_{i=1}^{n-1} L_{i}
$$

For polygons, triangle method is used to calculate area. When walking around the field once with GPS receiver, for example the triangle consists of apexes 1, 2, 3 (the shadow in diagram 1), the area is:

$$
S_{1}=\sqrt{p(p-a)(p-b)(p-c)}
$$

In the above equation:

$$
p=(a+b+c) / 2, a=\sqrt{x_{2}^{\prime 2}+y_{2}^{\prime 2}}, b=\sqrt{x_{3}^{\prime 2}+y_{3}^{\prime 2}}, c=L_{2}
$$

Then the area of field made of $\mathrm{n}$ points is:

$$
S=\sum_{i=1}^{n-2} S_{i}
$$

\section{MEASURING INSTRUMENT DESIGN}

GPS positioning information collecting and displaying system consists of microcontroller 89C51, LCD displaying module MGLS12864 and GPS module GARMIN GPS25LP shown as Figure 2. It can display Beijing Time, longitude \& latitude and ground horizontal height and other real time information. As the main controller, microcontroller controls GPS OEM board to receive, read and transfer data. GPS OEM board receives the signals sent from satellites; output them in NMEA 0183 format. After RS223Cand COMS/TTL electrical level transformation, output serial data are sent to the serial port of microcontroller. After processing, information to be displayed is selected by keyboard, finally sent to LCD MGLS12864, Liquid Crystal Display will update the content to be displayed in time.

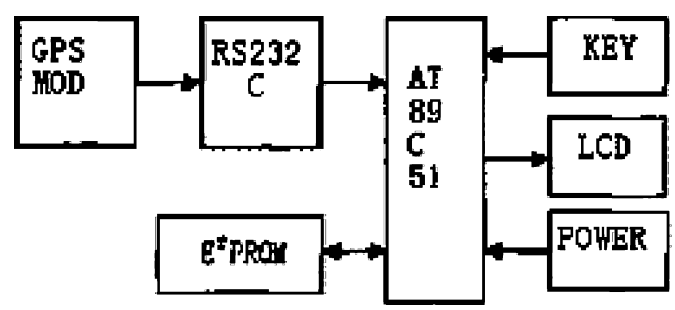

Figure 2. Block diagram of instrument

GPS module is applied to receive GPS satellite signals, output positioning information in NMEA format. System takes 25LP GPS OEM board made by 
GARMIN to realize positioning. This module integrates GPS receiver, interior antenna, electrify restoration circuit, signal interface circuits etc, $3.0-5.5 \mathrm{~V}$ wide range supplying electricity, 12 channel $5 \mathrm{~m}$ positioning accuracy. Microcontroller is the core of instrument. System adopts AT89C51 microcontroller as system processor, with high cost-performance, realizing data receiving, processing, displaying, storing and printing etc.

System working principles as follows: GPS module receives GPS satellite signals, outputs positioning information through RS-232 serial ports; communication interface transforms RS-232 serial signals into COMS/TTL electrical level and input them into the serial ports of microcontroller. Microcontroller processes positioning information under the control of software, receives orders from keyboard and display the geometric size calculation results on LCD or store them into serial E2PROM storage.

C51 language is used to write microcontroller software, and in the environment of Keil C51 compiling and debugging is carried out. Software mainly includes data receiving, calculating, keyboard scanning, printing module and etc. Microcontroller receives positioning information by serial port interruption method. Serial parameters are set: $4800 \mathrm{bps}, 8$ data bits, 1 stop bit, no check bit. Serial ports interruption is core software, flowchart is shown as Fig. 3.

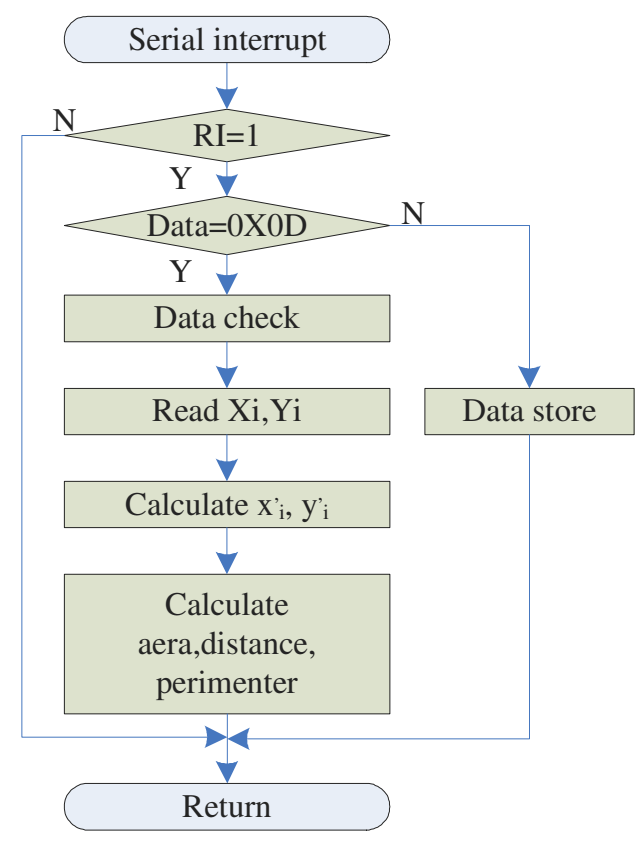

Figure 3. Flowchart of serial interrupt 


\section{EXPERIMENT AND ERROR ANALYSIS}

Experiment is carried out in the standard $400 \mathrm{~m}$ playground in school. According to first running track parameters, we can calculate that the area is $10695 \mathrm{~m} 2$. Walking around the first track when measuring, walking with normal speed in straightway and lower speed in curve way. Table 1 provides statistical data of 10 times measurements. LCD displays 3 standard playground outlines in different locations after 3 GPS positioning measurements. Though there is obvious deviation, they all keep good standard shape of playground, with similar area. It proves that low-cost GPS positioning has drift error relating to time, the accuracy is not good enough. However, in a short period, drift is not big, so the acquired track is basically similar, and the results are basically the same.

Table1. Statistics of experimental data

\begin{tabular}{|lc|}
\hline Standard playground \\
\hline Area/m2 & 10695 \\
\hline Times & 10 \\
\hline Mean value/m2 & 10567 \\
\hline Mean relative error $(\%)$ & 1.2 \\
\hline
\end{tabular}

Seen from outline, when moving fast, positioning points distribute sparsely, some key points are possibly missed. These missed points have big influence for calculating area. So in the curve parts, speed should be lowered down. With high dense points to measure, the key points can be ensured not missed. Measuring straight line has no big influence on area, so the speed can be high. Measuring statistics in Table 1 proves that relative error of measuring playground area is $1.2 \%$, which means GPS measuring instrument has good accuracy. Playground includes semicircle area prove that the instrument can well measure non-rectangle field area.

Hand-held GPS positioning error is mainly of system error and coarse error. Coarse error mainly comes from observation conditions, such as poor satellite visibility conditions; and system error mainly comes from GPS positioning satellites and atmospheric delay of positioning signal. Due to random error has characteristics as measuring times approaches infinity and expect value approaches 0 , so with mean value revised method to recognize and modify observation error, $2.5 \mathrm{~m}$ hang-held GPS positioning accuracy and area measuring error less than $2 \%$ is eventually acquired. 


\section{CONCLUSION}

In precision agriculture management, it is very necessary to fast real-time measure the geometric size of the field. Traditional measuring technology can hardly fast measure geometric size of irregular field and satisfy the requirements of precision agriculture. This design complete software and hardware development of portable instrument for measuring field geometric size. After practical testing and debugging, system runs normally. The development of this instrument provides a new device for fast measuring geometric size of fields. The main characteristic of the instrument is fast measuring geometric size of fields in any shape, with high measuring accuracy, providing accurate basis for managing production and agriculture machinery operation in precision agriculture.

\section{REFERENCES}

Zhang Nai-gian, Wang Mao-hua, Wang Ning. Precision agriculture-a worldwide overview [J] Computer sand Electronics in Agriculture, No. 3, 2002, pp. 113-132.

Zhu Yun-giang, Gong Hui-Ii, XU Hui-ping. Map projection transformation of GIS [J]. Journal of Capital Normal University, No. 22(3), 2001, pp. 88-94. 